\title{
Evolution of topological edge modes from honeycomb photonic crystals to triangular-lattice photonic crystals
}

\author{
Jin-Kyu Yang $\odot,{ }^{1,2,{ }^{*}}$ Yongsop Hwang, ${ }^{2}$ and Sang Soon Oh $\oplus^{3, \dagger}$ \\ ${ }^{1}$ Department of Optical Engineering, Kongju National University, Cheonan 31080, South Korea \\ ${ }^{2}$ Institute of Application and Fusion for Light, Kongju National University, Cheonan 31080, South Korea \\ ${ }^{3}$ School of Physics and Astronomy, Cardiff University, Cardiff CF24 3AA, United Kingdom
}

(Received 13 January 2021; revised 5 April 2021; accepted 5 May 2021; published 21 June 2021)

\begin{abstract}
The presence of edge modes at the interface of two perturbed honeycomb photonic crystals with $C_{6}$ symmetry is often attributed to the different signs of Berry curvature at the $\mathrm{K}$ and $\mathrm{K}^{\prime}$ valleys. In contrast to the electronic counterpart, the Chern number defined in photonic valley Hall effect is not a quantized quantity but can be tuned to a finite value including zero simply by changing geometrical perturbations. Here, we argue that the edge modes in photonic valley Hall effect can exist even when Berry curvature vanishes. We numerically demonstrate the presence of the zero-Berry-curvature edge modes in triangular-lattice photonic crystal slab structures in which $C_{3}$ symmetry is maintained but the inversion symmetry is broken. We investigate the evolution of the Berry curvature from the honeycomb-lattice slab structure to the triangular-lattice photonic crystal slab and show that the triangular-lattice photonic crystals still support edge modes in a very wide photonic band gap. We find that the edge modes with zero Berry curvature can propagate with extremely low bending loss along the interface formed by the triangular-lattice photonic crystals.
\end{abstract}

DOI: 10.1103/PhysRevResearch.3.L022025

\section{INTRODUCTION}

Topological insulators, which are insulating in the bulk region while conductive along the edge, have been intensively studied due to their intriguing physical properties as well as potential applications [1-3]. As an optical counterpart of the topological insulators in condensed matter physics, photonic topological insulators (PTIs) have been proposed and demonstrated with gyro-optic materials in microwave region [4-6] and with coupled helical waveguides at visible wavelengths [7]. PTIs show unique characteristics, for example, the guided modes along the edge or interface of PTIs, which are robust against defects and deformations due to topological protection [8-11]. Such robustness has been experimentally demonstrated at telecommunication wavelengths [12,13].

Recently, the optical quantum spin Hall effect (QSHE) and optical quantum valley Hall effect (QVHE) have been realized by introducing geometrical perturbation in PTIs such as a honeycomb (HC) photonic crystal (PhC) structure. For instance, for the QSHE PTIs [14-16], a photonic band gap (PBG) can be created at Dirac point by extending or shrinking while the $C_{6}$ symmetry is maintained. In this breathing $\mathrm{HC}-\mathrm{PhC}$, the pseudo-time-reversal symmetry is protected and pseudo-spin

\footnotetext{
*jinkyuyang@kongju.ac.kr

†OhS2@cardiff.ac.uk
}

Published by the American Physical Society under the terms of the Creative Commons Attribution 4.0 International license. Further distribution of this work must maintain attribution to the author(s) and the published article's title, journal citation, and DOI. channels are maintained (pseudo-time-reversal operator $\mathcal{T}$ is defined to satisfy $\mathcal{T}^{2}=-1$ ) [14]. Recently, the robustness of corner states in the same structure was shown to stem from an obstructed atomic limit phase [17]. On the other hand, for the QVHE PTIs, inversion symmetry breaking opens a PBG as in a staggered HC lattice $[18,19]$ and a perturbed kagome lattice [20]. For both QSHE and QVHE PTIs, edge modes, which are localized at the interface between two PTIs with different signs of perturbation, exist inside the PBGs.

Although the near $100 \%$ transmission has been shown using the edge modes in the optical QVHE [18-20], the origin of the edge modes is not clearly understood because they are not topologically protected in the same way as in the Chern insulators (the Chern number is zero for optical QVHEs). One way of explaining the existence of edge modes in the optical QVHE is the bulk-edge correspondence in an extended parameter space $\left(k, a_{g}\right)$ where $k$ is the wave vector and $a_{g}$ is the perturbation strength [21]. Another most common explanation is the valley degree of freedom, which originates from the different signs of Berry curvature for different valleys $[11,18]$. However, it is still unclear whether the different signs of Berry curvature for different valleys is a necessary condition for the existence of the edge modes and reflection-less propagation at the bending.

In this paper, we report that edge modes can be created even when the Berry curvature vanishes. We demonstrate this by studying the Berry curvature of photonic bands for $\mathrm{PhC}$ slabs with the staggered $\mathrm{HC}$ lattices including the $\mathrm{HC}$ lattice and the triangular lattice. We show that the triangular-PhC (Tri-PhC) is an extreme case of the staggered HC-PhCs that holds their topological characteristics because the reduction of one hole in the unit cell to null leads to the Tri-PhCs. 

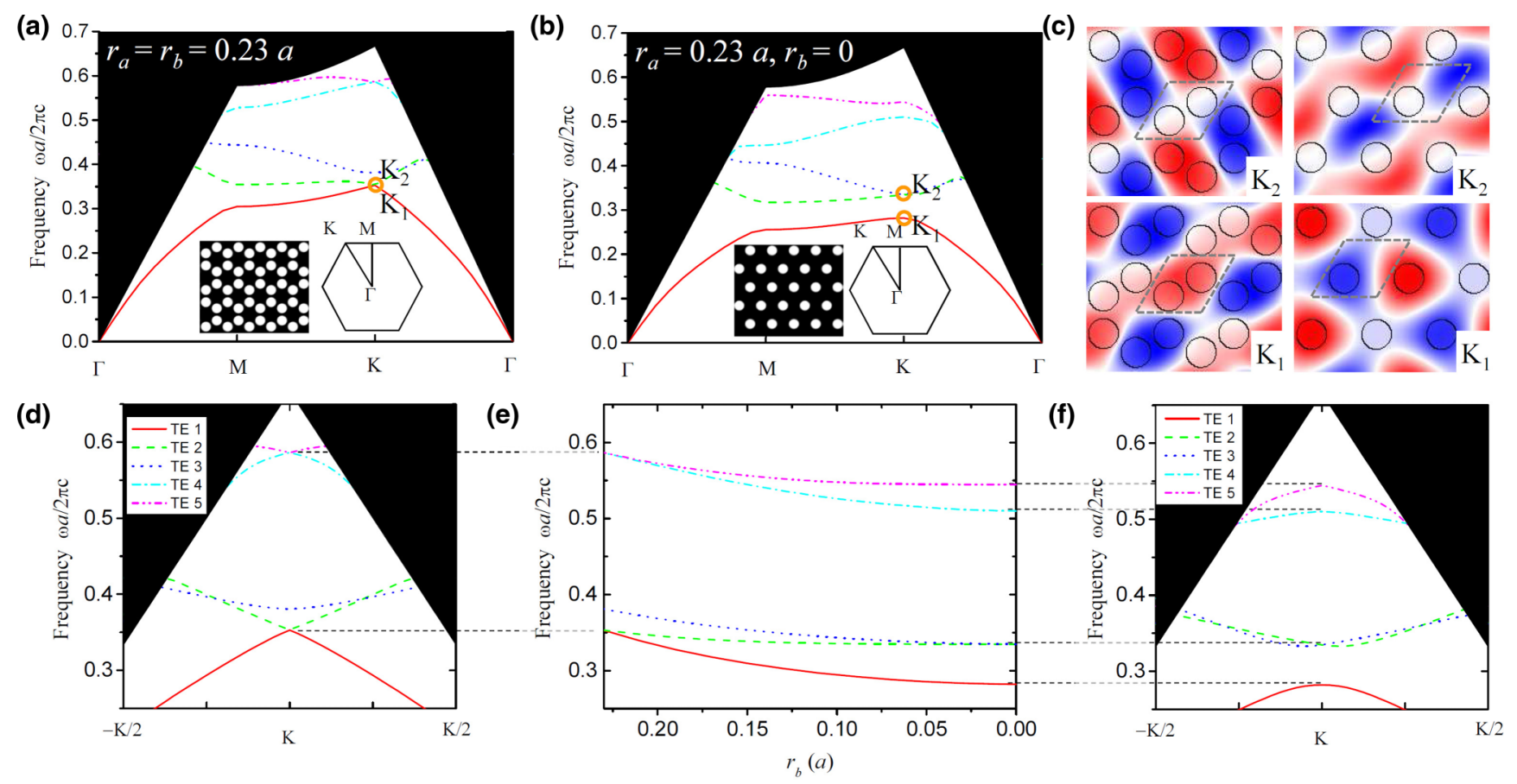

FIG. 1. Characteristics of the TE photonic band structures. (a) Band diagram of the air-hole HC-PhC slab structure with $r_{a}=r_{b}=0.23 a$ (b) Band diagram of the air-hole Tri-PhC slab structure with $r_{a}=0.23 a\left(r_{b}=0\right)$ (c) Magnetic field profiles of the normal component $\left(H_{z}\right)$ at the center of the slab. $\mathrm{K}_{1}, \mathrm{~K}_{2}$ mean the first and the second $\mathrm{K}$-point TE modes shown in (a) and (b). (d) Band diagram near the $\mathrm{K}$ point at HC-PhC slab. (e) Frequency of the K-point TE modes as a function of $r_{b}$. (f) Band diagram near the K point at the Tri-PhC slab

This argument is supported by the numerical calculation of the photonic band structures and Chern numbers and the simulation of one-way propagation of the edge modes.

\section{PHOTONIC BAND STRUCTURE ANALYSIS}

First, we perform the photonic band structure analysis to understand the characteristics of the guided modes in the staggered HC-PhC slab structure composed of two air holes in its unit cell. The structure becomes a HC-PhC slab [the inset of Fig. 1(a)] if the radii of the two holes are identical and becomes a Tri-PhC slab [the inset of Fig. 1(b)] if one of the air holes is missing. Therefore, the geometrical transition from the HC-PhC to the Tri-PhC can be described by the change of the radius of the smaller air hole $\left(r_{b}\right)$ from $0.23 a$ to zero.

Figure 1(a) shows the band diagram of the HC-PhC slab $\left(r_{b}=0.23 a\right)$ calculated by the three-dimensional (3D) planewave expansion method [22]. Here, the refractive index of the slab is set to 3.16 , and the radius of the large air hole $\left(r_{a}\right)$ and the thickness of the slab $(t)$ are fixed as $0.23 a$ and $0.47 a$, respectively. At the $\mathrm{K}$ point in the band diagram of the $\mathrm{HC}-\mathrm{PhC}$, one can observe the Dirac cone, where the lowest and the second-lowest TE bands meet with a linear slope. In general, the Dirac cone exists when there are $C_{3}$ symmetry and the inversion symmetry with respect to the mid-point of two air holes in the unit cell (HC lattice) [23].

Figure 1(b) shows the band diagram of the Tri-PhC structure $\left(r_{b}=0\right)$. When the inversion symmetry is broken (a staggered HC lattice), the degeneracy at the Dirac cone is lifted opening a PBG where a one-way propagation mode can be introduced at the interface of two PTIs with different signs of geometric perturbation. The evolution of the band diagram by reducing the radius of one air hole is shown in Figs. 1(d), 1(e), and 1(f). As the radius of the one air hole decreases, the PBG is opened at K point because of the inversion symmetry breaking and becomes wider until the smaller air hole is completely removed.

The PBG opening can be understood by looking at the electromagnetic field profiles of the lowest and the secondlowest TE bands. When the inversion symmetry is maintained $\left(r_{a}=r_{b}\right)$, the magnetic fields are localized equally at both holes in a single unit cell [Fig. 1(c)] resulting in a degeneracy at $\mathrm{K}$ point as shown in Fig. 1(a). However, the inversion symmetry breaking $\left(r_{a}>r_{b}\right)$ makes the magnetic field distribution asymmetric with respect to the midpoint of two holes for the first and second band edge modes $\left(\mathrm{K}_{1}\right.$ and $\left.\mathrm{K}_{2}\right)$. This can be clearly seen in Fig. S1 within the Supplemental Material [24] where the magnetic field is localized around larger holes for $\mathrm{K}_{1}$ in the first band of staggered HC-PhCs while the magnetic field is localized around smaller holes for $\mathrm{K}_{2}$ in the second band (see Supplemental Material [24] for more details). Remarkably, the overall field distributions of the two band edge modes are maintained until $r_{b}=0$ (Tri-PhC) which has the maximum $\mathrm{PBG}$ at the $\mathrm{K}$ point as shown in Fig. 1(c). Note that the PBG is open even with a very small difference between $r_{a}$ and $r_{b}$ because the PBG originates from the geometrical inversion symmetry breaking.

\section{BERRY CURVATURE AND VALLEY CHERN NUMBER}

In order to verify the optical QVHE in the $\mathrm{HC}-\mathrm{PhCs}$ with nonidentical air holes, we investigate the evolution of the Berry curvature of the first band and its half Chern number from $r_{b}=0$ to $r_{b}=r_{a}$. The Berry curvature 
(a)

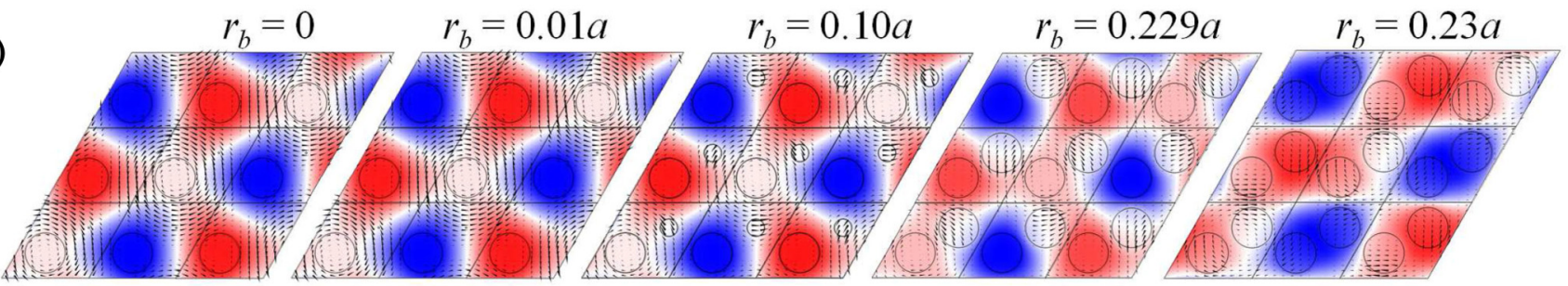

(b)

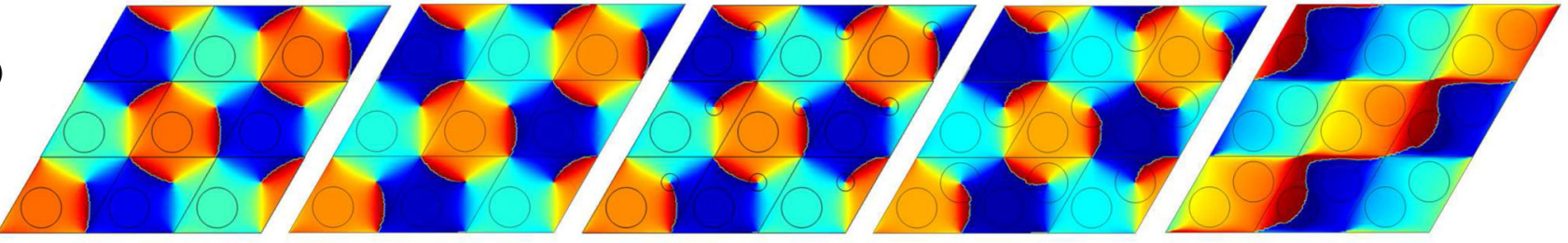

(c)

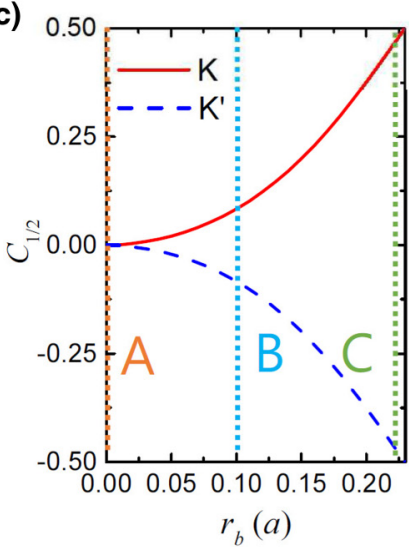

(d)

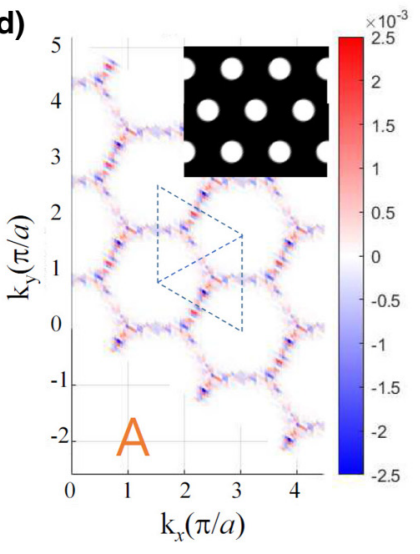

(e) 5

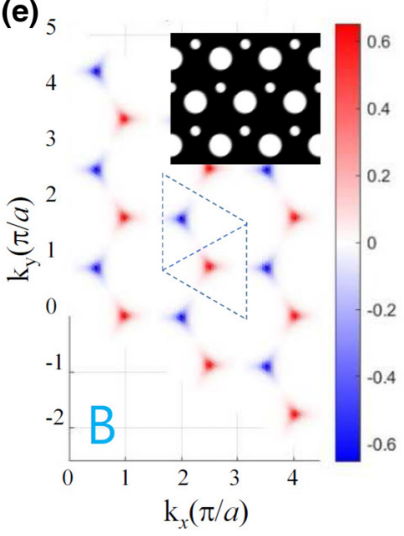

(f)

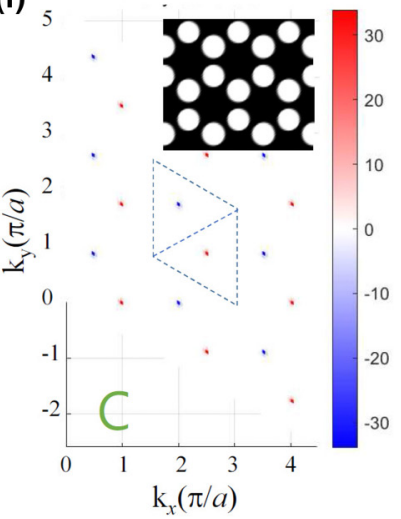

FIG. 2. (a) The normal magnetic field $\left(H_{z}\right)$ profiles of the first TE mode at the K point with the in-plane electric field vectors $\left(\vec{E}_{\|}\right)$as the structure is gradually changed from the Tri-PhC $\left(r_{b}=0\right)$ to the HC-PhC $\left(r_{b}=0.23 a\right)$ and (b) the phase profile of $H_{z}$. (c) The half Chern number integrated in the half of the first Brillouin zone as marked in (d) and (e) with dashed lines. [(d), (e)] Berry curvatures of the first TE band (d) $r_{b}=0$ (A) (e) $r_{b}=0.10 a(\mathrm{~B})$, and (f) $r_{b}=0.229 a$ (C, slightly perturbed HC-PhCs).

$\mathcal{F}(\mathbf{k})=\nabla_{\mathbf{k}} \times i\left\langle\mathbf{E}_{\mathbf{k}} \mid \nabla_{\mathbf{k}} \mathbf{E}_{\mathbf{k}}\right\rangle$ is numerically calculated by summing up the phases of the electric fields $\mathbf{E}_{\mathbf{k}}$ at the four points of the plaquette in the discretized $\mathbf{k}$ space. Then the valley Chern number $C_{v}$ is given as $C_{v}=C_{1 / 2, \mathrm{~K}}-C_{1 / 2, \mathrm{~K}^{\prime}}$ where $C_{1 / 2, \mathrm{~K}}$ $\left(C_{1 / 2, \mathrm{~K}^{\prime}}\right)$ is the half Chern number calculated by integrating the Berry curvature over the triangular area around the $\mathrm{K}$ $\left(\mathrm{K}^{\prime}\right)$ point $[25,26]$. Here, we consider two-dimensional (2D) cylindrical air-hole $\mathrm{PhCs}$ because the field profile of the TE mode calculated by $2 \mathrm{D}$ calculations is the same as the $3 \mathrm{D}$ calculation except the variation along the plane-normal direction [27]. In consideration of the finite thickness of the PhC slab, the refractive index of dielectric materials in $2 \mathrm{D}$ calculation is set to 2.4 .

Figures 2(a) and 2(b) show the normal magnetic field component $\left(H_{z}\right)$ profiles of the first TE mode at the $\mathrm{K}$ point, and its phase change. The arrows in Fig. 2(a) indicate the in-plane electric field vectors. At $r_{b}=0$ (Tri-PhC), the magnetic field is localized near the large air holes with the radius $r_{a}$, and the localization behavior is preserved unless $r_{b}=r_{a}$. When the inversion symmetry is broken, there are two vortices: one at the center of hexagons and the other at the center of smaller holes. In the amplitude plots [Fig. 2(a)], the amplitude becomes zero at the two vortices. In the phase plots [Fig. 2(b)], two vortices show different signs. This relation has been mathematically proved in a rigorous manner [25]. The optical vortices with different signs, which are chiral, can be observed in the in-plane electric field vector as shown in Fig. 2(a). The chirality of the optical vortices in both magnetic and electric field is related to the photonic valleys, similarly to the valley pseudospin in an electronic system, thereby the photonic valley modes can be selectively excited by chirality of the circularly polarized electromagnetic field [10]. This means that the photonic valley modes (edge modes) can be manipulated by the chiral vortex-valley locking, i.e., the circularly polarized light source excitation at the singularity points (for example, the center of large air holes in the interface). We noted that a similar explanation was made for the edge modes in Tri-PhCs [28].

Interestingly, the Berry curvature in the momentum (k) space becomes zero as $r_{b}$ approaches zero (Tri-PhC) leading to the zero half Chern as shown in Figs. 2(c)-2(f). Nevertheless, the two vortices with different signs still remain when $r_{b}=0$, which implies that topologically protected modes could exist in the interface or edge within a wide spectral range of PBG of the Tri-PhCs (see Figs. S2, S3, and S4 within the Supplemental Material [24]). Indeed, topological edge modes exist as we will show numerically in the next section. Recently, it was reported that the photonic 2D 
(a)

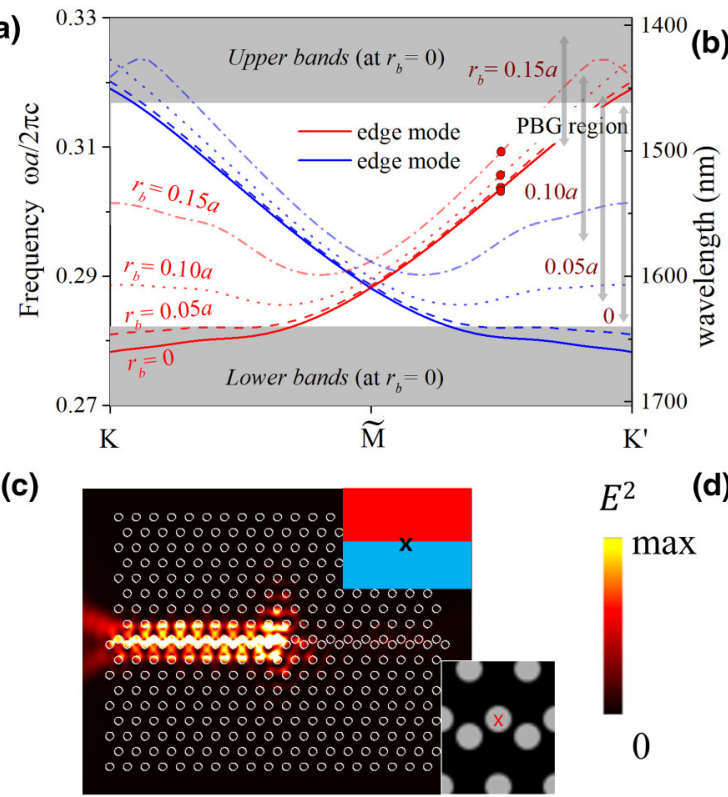

(b)

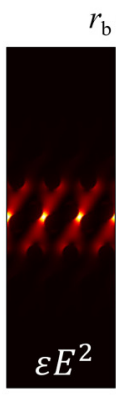

$r_{\mathrm{b}}=0$

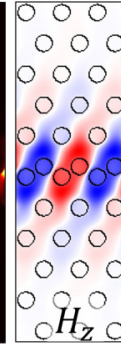

$0.05 a$

$0.10 a$

$0.15 a$

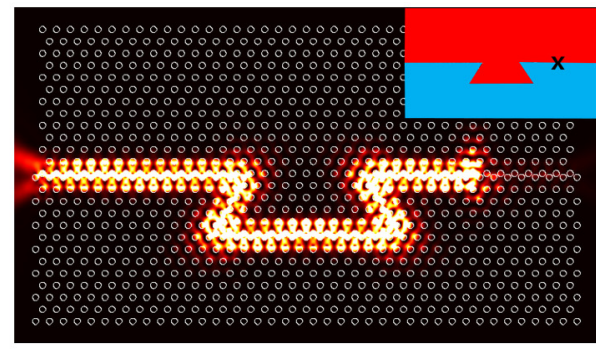

FIG. 3. Analysis of photonic topological edge modes. (a) Dispersion properties of the edge modes in the PTIs with various $r_{b}$. The gray region indicates the bulk bands. The white region (or the gray arrows at the right) indicates the PBG region of the given $r_{b}$. (b) $H_{z}$ field profiles of the edge modes in the PTIs with various $r_{b}$ as marked with red circles at (a). (c) Time-averaged electric field intensity distribution of the edge mode at $\lambda=1550 \mathrm{~nm}$ excited at the center of air holes in the straight interface as shown in the inset with the red mark. (d) Time-averaged electric field intensity distribution in the $\mho$-shape interface. The marks, $x$ in the insets of (c) and (d) indicate the position of the excited clock-wise chiral source.

Su-Schrieffer-Heeger (SSH) lattice inherently has the $C_{4 v}$ point group symmetry and zero Berry curvature $[29,30]$. According to Wakabayashi's report, the nontrival topological properties with zero Berry curvature can be realized by the curl of the magnetic field [29]. Our numerical results suggest that the existence of topological edge modes in Tri-PhCs and even in staggered $\mathrm{HC}$ lattices, which have the $C_{3}$ symmetry, may not be related to the Berry curvatures with different signs. We believe that more theoretically rigorous work will clarify the relationship between the vortices at valleys and the topological protected states.

\section{ONE-WAY PROPAGATION}

Given the continuity of the evolution of the band diagram, it is obvious that the Tri- $\mathrm{PhC}$ is one extreme case of the PTIs based on the staggered HC-PhCs. Accordingly, the Tri-PhCs are expected to have topologically protected edge modes, which are the same kind as the ones in the staggered HC-PhCs. As a result of the chiral vortex-valley locking, the topologically projected edge modes are chiral implying that the two counter-propagating modes can be selectively excited by chiral sources with different chiralities as we will see in this section. Hence, we designed a structure of a pair of the HC-PhCs with nonidentical air holes, and one of them is vertically flipped and laterally shifted to form an interface between them as shown in Fig. 3. To find guided modes along the interface, we calculated a band diagram in Fig. 3(a) showing the existence of interface modes. The two guided modes in the PTIs with various $r_{b}$ are found in the PBGs, which are denoted by the gray arrows at the right side of Fig. 3(a). Figure 3(b) shows the amplitude distributions of the magnetic field of the edge modes marked with red circles in Fig. 3(a). Because of the wide PBG, the edge mode in the Tri-PhCs $\left(r_{b}=0\right)$ is strongly localized at the interface. However, as $r_{b}$ increases, the PBG becomes narrower and the localization of the edge mode in the interface becomes weaker.

We also investigated the one-way propagation properties of the edge mode in the Tri-PhC by the 3D finite-difference time-domain (FDTD) method [30]. In case of $r_{b}=0$ (Tri-PhC slab), it is clear that the edge mode propagates unidirectionally along the straight interface as shown in Fig. 3(c). Here, the mode is excited by the clockwise $(\mathrm{CW})$ chiral source generated by two dipole sources with $\pi / 2$ phase difference at $\lambda=1550 \mathrm{~nm}$. In Fig. 3(c), the upper inset shows a schematics of a pair of Tri-PhC slab, and the lower shows the position of the chiral source with the red $\times$ mark. Even in the $\mho$-shape interface with four $120^{\circ}$ bending geometry, the edge mode propagates along the interface without reflection near the sharp corners shown in Fig. 3(d).

For the quantitative analysis of chiral coupling of the guided mode along the PTI interface, we calculated one-way coupling efficiency defined as the ratio of the left (or right) propagating flux to both the left and the right propagating energy fluxes. Here, the $\mathrm{CW}$ chiral source was excited at the center of air holes, and the time-averaged Poynting flux monitored at the left (or right) edge of the photonic crystals was obtained for energy flux. Figure 4(a) shows one-way coupling efficiency as a function of wavelength of the $\mathrm{CW}$ chiral source excited at the center of the straight interface. The blue indicates the coupling efficiency of the $\mathrm{CW}$ chiral source into the propagating mode toward the left direction. Because of the wide PBG of the Tri-PhCs, high one-way coupling efficiency over $90 \%$ is found in a broad spectral range from $1490 \mathrm{~nm}$ 

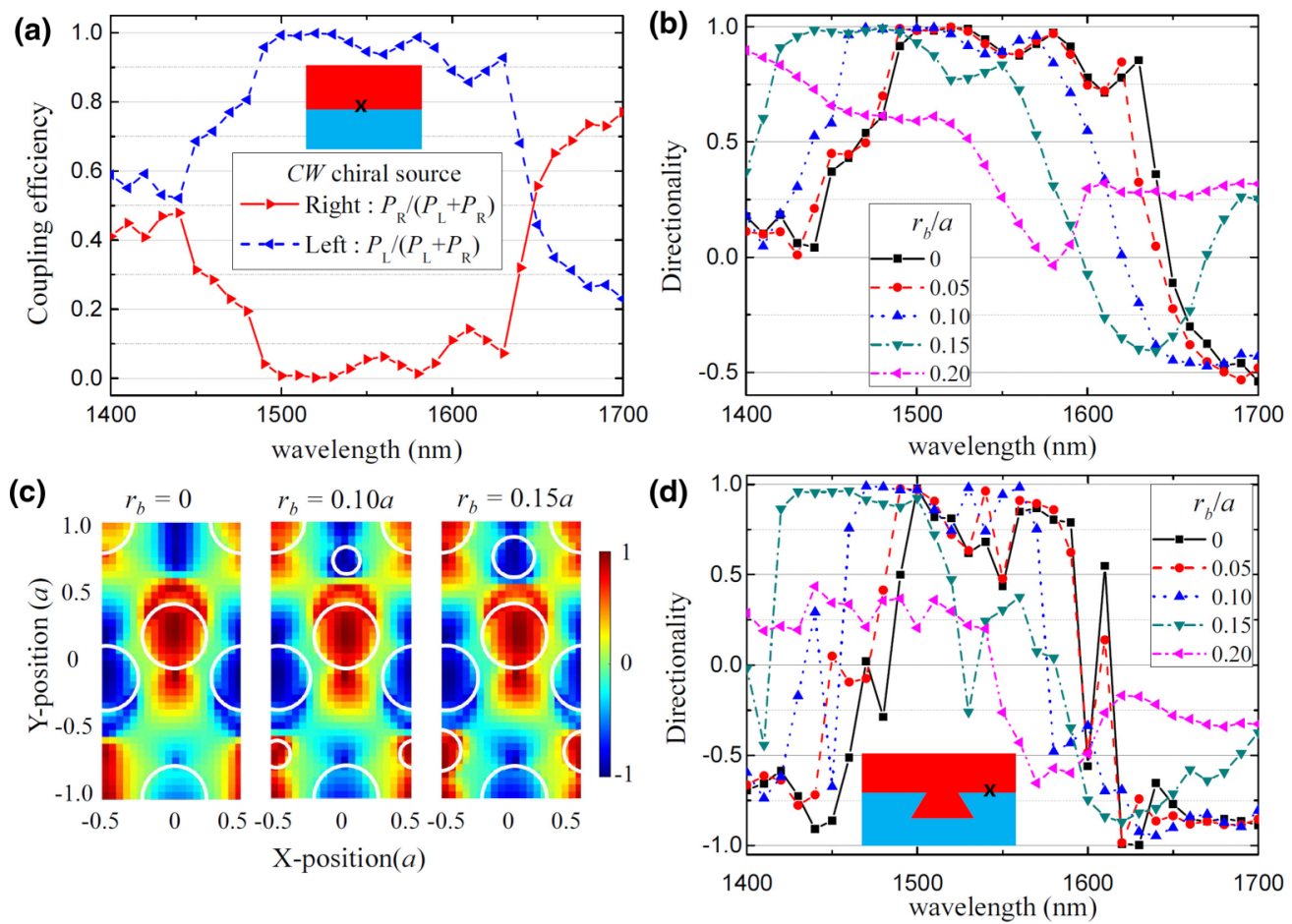

FIG. 4. Characteristics of the one-way propagating edge modes. (a) One-way coupling efficiency of the CW chiral source into the edge modes in the straight interface with $r_{b}=0$ (Tri-PhC slabs). (b) Directionality of the excited edge modes in the straight interface with various $r_{b}$. (c) The source-position dependent directionality with various $r_{b}$ at the CW chiral source. (d) Directionality of the excited edge modes in the $\mho$-shape interface with various $r_{b}$.

to $1600 \mathrm{~nm}$ as shown in Fig. S5 within the Supplemental material [24]. For the systematical analysis for the unidirectional coupling efficiency of the edge propagation, we defined the directionality as $\left(P_{\mathrm{L}}-P_{\mathrm{R}}\right) /\left(P_{\mathrm{L}}+P_{\mathrm{R}}\right)$ at the $\mathrm{CW}$ chiral source [18]. Figure 4(b) shows the directionality in the HC-PhCs with nonidentical air holes from $r_{b} / a=0$ to $r_{b} / a=0.20$ as a function of the wavelength. In case of $r_{b}=$ 0 (Tri-PhC slabs), the spectral range of directionality over 0.90 is about $60 \mathrm{~nm}$. This wide spectral response of the directionality is very robust to the $r_{b}$. Especially, at $r_{b} / a=$ 0.1 , the flat top directionality over 0.98 is about $50 \mathrm{~nm}$. The blueshift of the frequency range of high directionality is owing to the blueshift of the PBG range as shown in Fig. 1(e). The pictures in Fig. 4(c) indicate the sourceposition dependent directionality in the $\mathrm{HC}-\mathrm{PhCs}$ with various $r_{b}$ when the $\mathrm{CW}$ chiral source was excited near the interface. In the simulation, we fixed the wavelength of the excited source as $1500 \mathrm{~nm}, 1470 \mathrm{~nm}$, and $1440 \mathrm{~nm}$ for $r_{b} / a=0$, 0.1 , and 0.15 , respectively, for the the highest directionality at Fig. 4(b). Here, the white lines indicate the air-hole boundary. When the point-like chiral source lies inside the upper large air hole $\left(r_{a}\right)$, the unidirectional propagation occurs along the left, however, the opposite directional propagation also observed when the source lies inside the lower large air hole $\left(r_{a}^{\prime}\right)$. The directional propagation occurs dominantly when the source is located at the air holes near the interface. Especially, the center of the upper large air hole in the interface is the optimal position of the $\mathrm{CW}$ point-like chiral source to achieve the perfect directional coupling along the left direction. In contrast, the optimal position for the perfect right directional coupling is the center of the lower large air hole in the interface. It implies that the unidirectionality is related with both the geometry of the large air hole and the chirality of the source. The asymmetric structural unidirectionality was also reported in the glide $\mathrm{PhC}$ waveguide structure [31].

To demonstrate the robustness of unidirectional topological transport along the interface without reflection at a sharp bending, we investigated directionality in the $\mho$-shape interface with various $r_{b}$. Figure 4(d) shows the directionality as a function of wavelength of the $\mathrm{CW}$ chiral source at the right side of $\mho$-shape interface. The spectral range of directionality over 0.8 is observed over $60 \mathrm{~nm}$, which is reduced in comparison with the straight interface. However, at the optimal wavelength the directionality in the $\mho$-shape interface is almost same as that in the straight interface. For example, in case of $r_{b}=0.10 a$, the directionality at $\lambda=1470 \mathrm{~nm}$ in the straight interface and the $\mho$-shape interface is $0.995,0.989$, respectively. This indicates that the topological transport is robust with reflection at the sharp bending corner, at least for the optimal condition. The sudden decrease of the directionality at the longer wavelength is due to the band-edge behaviors of the edge mode (see Supplemental Material [24]).

\section{CONCLUSIONS}

We have numerically demonstrated the evolution of the topological behavior from the perfect $\mathrm{HC}$ slab to the Tri-PhC slab. When one of the two air holes in the unit cell of the $\mathrm{HC}$ lattice gradually decreases, the Dirac cone at the K point disappears and the photonic band gap opens because of inversion 
symmetry breaking. From the investigation of the evolution of the Berry curvature from the $\mathrm{HC}-\mathrm{PhC}$ to the Tri-PhC, we have shown that the topological edge modes exist and one-way propagation of the edge modes are maintained even with zero Berry curvature at the Tri-PhC, which is explained by the chiral vortex-valley locking. We believe that our analysis of the staggered $\mathrm{HC}-\mathrm{PhC}$ slab will be useful for design of highly efficient platform of lossless photonic integrated circuits.

\section{ACKNOWLEDGMENTS}

This work was supported by the National Research Foundation of Korea (NRF) grant funded by the Korea government (MSIT) (Grants No. 2017R1A2B4012181 and No. 2020R1A2C1014498). This work is part-funded by the European Regional Development Fund through the Welsh Government [Grant No. 80762-CU145 (East)].
[1] C. L. Kane and E. J. Mele, Quantum Spin Hall Effect in Graphene, Phys. Rev. Lett. 95, 226801 (2005).

[2] B. A. Bernevig, T. L. Hughes, and S.-C. Zhang,Quantum spin Hall effect and topological phase transition in HgTe quantum wells, Science 314, 1757 (2006).

[3] M. Z. Hasan and C. L. Kane, Colloquium: Topological insulators, Rev. Mod. Phys. 82, 3045 (2010).

[4] F. D. M. Haldane and S. Raghu, Possible Realization of Directional Optical Waveguides in Photonic Crystals with Broken Time-Reversal Symmetry, Phys. Rev. Lett. 100, 013904 (2008).

[5] Z. Wang, Y. D. Chong, J. D. Joannopoulos, and M. Soljacic, Reflection-Free One-Way Edge Modes in a Gyromagnetic Photonic Crystal, Phys. Rev. Lett. 100, 013905 (2008).

[6] A. B. Khanikaev, S. H. Mousavi, W.-K. Tse, M. Kargarian, A. H. MacDonald, and G. Shvets, Photonic topological insulators, Nat. Mater. 12, 233 (2013).

[7] M. C. Rechtsman, J. M. Zeuner, Y. Plotnik, Y. Lumer, D. Podolsky, F. Dreisow, S. Nolte, M. Segev, and A. Szameit, Photonic Floquet topological insulators Nature (London) 496, 196 (2013)

[8] X. Cheng, C. Jouvaud, X. Ni, S. H. Mousavi, A. Z. Genack, and A. B. Khanikaev, Robust reconfigurable electromagnetic pathways within a photonic topological insulator, Nat. Mater. 15, 542 (2016).

[9] W.-J. Chen, S.-J. Jiang, X.-D. Chen, J.-W. Dong, and C. T. Chan, Experimental realization of photonic topological insulator in a uniaxial metacrystal waveguide, Nat. Commun. 5, 5782 (2014).

[10] X.-D. Chen, F.-L. Zhao, M. Chen, and J.-W. Dong, Valleycontrasting physics in all-dielectric photonic crystals: Orbital angular momentum and topological propagation, Phys. Rev. B 96, 020202(R) (2017).

[11] T. Ma and G. Shvets, All-Si valley-Hall photonic topological insulator, New J. Phys. 18, 025012 (2016).

[12] M. I. Shalaev, W. Walasik, A. Tsukernik, Y. Xu, and N. M. Litchinitser, Robust topologically protected transport in photonic crystals at telecommunication wavelengths, Nat. Nanotechnol. 14, 31 (2019).

[13] S. Arora, T. Bauer, R. Barczyk et al., Direct quantification of topological protection in symmetry-protected photonic edge states at telecom wavelengths, Light: Sci. Appl. 10, 9 (2021).

[14] L.-H. Wu and X. Hu, Scheme for Achieving a Topological Photonic Crystal by Using Dielectric Material, Phys. Rev. Lett. 114, 223901 (2015).

[15] Y. Yang, Y. F. Xu, T. Xu, H. X. Wang, J. H. Jiang, X. $\mathrm{Hu}$, and Z. H. Hang, Visualization of a Unidirectional Electromagnetic Waveguide Using Topological Photonic Crystals Made of Dielectric Materials, Phys. Rev. Lett. 120, 217401 (2018).
[16] N. Parappurath, F. Alpeggiani, L. Kuipers, and E. Verhagen, Direct observation of topological edge states in silicon photonic crystals: Spin, dispersion, and chiral routing, Sci. Adv. 6 , eaaw4137 (2020).

[17] M. Proctor, P. A. Huidobro, B. Bradlyn, M. B. de Paz, M. G. Vergniory, D. Bercioux, and A. Garcia-Etxarri, Robustness of topological corner modes in photonic crystals, Phys. Rev. Research 2, 042038(R) (2020).

[18] X. T. He, E. T. Liang, J. J. Yuan, H. Y. Qiu, X. D. Chen, F. L. Zhao, and J. W. Dong, A silicon-on-insulator slab for topological valley transport, Nat. Commun. 10, 872 (2019).

[19] M. J. Mehrabad, A. P. Foster, R. Dost, E. Clarke, P. K. Patil, A. M. Fox, M. S. Skolnick, and L. R. Wilson, Chiral topological photonics with an embedded quantum emitter, Optica 7, 1690 (2020)

[20] S. Wong, M. Saba, O. Hess, and S. S. Oh, Gapless unidirectional photonic transport using all-dielectric kagome lattices, Phys. Rev. Research 2, 012011(R) (2020).

[21] The MATLAB codes used to calculate Berry curvature and Chern numbers are available on https://github.com/PTIcode/ ChernCalc.

[22] M. Saba, S. Wong, M. Elman, S. S. Oh, and O. Hess, Nature of topological protection in photonic spin and valley Hall insulators, Phys. Rev. B 101, 054307 (2020).

[23] S. G. Johnson and J. D. Joannopoulos, Block-iterative frequency-domain methods for Maxwell's equations in a planewave basis, Opt. Express 8, 173 (2001).

[24] See Supplemental Material at http://link.aps.org/supplemental/ 10.1103/PhysRevResearch.3.L022025 for band analysis and field analysis of photonic topological edge modes.

[25] COMSOL Multiphysics v. 5.4. www.comsol.com. COMSOL AB, Stockholm, Sweden.

[26] J. L. Liu, W. M. Ye, and S. Zhang, Pseudospin-induced chirality with staggered optical graphene, Light: Sci. Appl. 5, e16094 (2016).

[27] F. Liu and K. Wakabayashi, Novel Topological Phase with a Zero Berry Curvature, Phys. Rev. Lett. 118, 076803 (2017).

[28] F. Liu, H. Y. Deng, and K. Wakabayashi, Topological photonic crystals with zero Berry curvature, Phys. Rev. B 97, 035442 (2018).

[29] D. J. Bisharat and D. F. Sievenpiper, Topological local-valley interface in ordinary photonic crystal waveguide, Proc. SPIE 11290, 112900N (2020).

[30] A. Taflove and S. G. Hagness, Computational Electrodynamics: The Finite-Difference Time-Domain Method (Artech House, Boston, 2005).

[31] I. Söllner, S. Mahmoodian, S. L. Hansen, L. Midolo, A. Javadi, G. Kiršanske, T. Pregnolato, H. El-Ella, E. H. Lee, J. D. Song, S. Stobbe, and P. Lodahl, Deterministic photon-emitter coupling in chiral photonic circuits, Nat. Nanotechnol. 10, 775 (2015). 